\title{
ÁRABE PARA TODOS. EL DESAFÍO DE ELABORAR MATERIALES PARA LA ENSEÑANZA DEL ÁRABE MARROQUí
}

\author{
Victoria AGUILAR ${ }^{*}$ \\ Universidad de Murcia
}

BIBLID [1133-8571] 26 (2019) 14.1-16.

Resumen: La realidad lingǘstica de la LA (lengua árabe) pone en evidencia la convivencia simultánea de, al menos, dos variedades. La competencia en los dos registros similar a la de un nativo (digamos C1) sería la prueba indiscutible para acreditar un dominio en árabe. En el contexto español, la enseñanza de la LA implica necesariamente la enseñanza del marroquí o dariya. Además, el uso del árabe marroquí se expande a campos que antes le estaban restringidos. Uno de los desafíos más acuciantes que se plantea en el ámbito de la enseñanza del dariya es la elaboración de materiales. Para garantizar el éxito de la empresa, es ineludible acometerla siguiendo las directrices de los presupuestos actuales en el ámbito de segundas lenguas.

Palabras clave: Enseñanza del árabe como lengua extranjera (EALE), Enfoque integrado, Árabe marroquí.

Abstract: «Arabic for all. The challenge of developing materials for teaching Moroccan Arabic». A linguistic approach to the Arabic language demonstrates that at least two simultaneous varieties coexist. More and more, proving proficiency in Arabic requires competence in both registers. In the Spanish context, the teaching of Arabic language should necessarily mean the teaching of Moroccan Arabic or Darija. Besides, Moroccan Arabic is spreading its use to fields that were previously "forbidden". One of the current challenges is the development of materials for teaching darija. To ensure success, it is important to follow the guidelines of current research in the field of L2.

Key words: Teaching Arabic as a Foreign Language (TAFL), Integrated approach, Moroccan Arabic.

Profesora titular de Lengua Árabe. E-mail: aguilar@um.es 


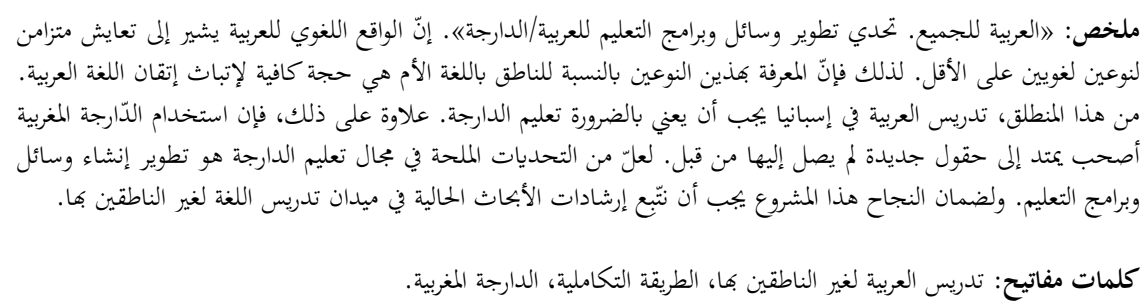

\section{Introducción}

Durante décadas, la LA (Lengua Árabe) se enseñó como si fuera una lengua muerta, de forma semejante al latín o griego clásicos, sin prestar mucha atención a las variantes locales ni a su realidad como instrumento oral de comunicación. En España, saber árabe servía para acceder a los textos escritos, por lo que aprender la lengua significaba conocer las reglas gramaticales para leer cierto tipo de textos. El auge de los métodos comunicativos, de los enfoques por tareas y otros avances en el campo de la enseñanza de lenguas extranjeras produjo un despliegue de métodos y materiales, que se centraron exclusivamente en la adquisición del árabe estándar. Es decir, la lengua meta pasó de ser el árabe clásico a ser el árabe estándar moderno (Versteegh 2006). Sin embargo, la mayoría de estas nuevas iniciativas fracasaron al no considerar un registro oral real de la lengua (Wahba 2006: 140-141), con secuelas muy desalentadoras para los alumnos. Los resultados de aprendizaje no eran equiparables con los esfuerzos empleados, lo que producía una frustración cada vez mayor (Aguilar \& Fernández: 2016, Palmer 2007 y Wahba 2006: 144).

Como en otros muchos aspectos, Estados Unidos fue pionero en simultanear en el mismo espacio y tiempo la enseñanza de la LA estándar con una variante regional, de forma similar a cómo ocurría en la vida cotidiana. El profesor Munther Younes de la universidad de Cornell fue uno de los iniciadores hace más de treinta años ya, de la metodología denominada «árabe integrado» (Younes 2104). Sucesivamente, muchas otras universidades comenzaron a implementar programas similares, en los que se incluían las variantes regionales, bien siguiendo la misma metodología o con algunas variantes. Atendiendo a su rentabilidad, los registros más frecuentes han sido el árabe egipcio y el levantino. Actualmente ya se admite que para ser competente 
en árabe es necesario serlo en al menos dos registros (Alosh 1997 y al-Batal 2006).

En el caso de España, por razones obvias, se imponía aplicar esta nueva tendencia utilizando el árabe estándar con el árabe marroquí o dariya (Aguilar 2014). A nadie se le escapa que las dificultades en este caso son mucho mayores, ya que el árabe levantino y el estándar comparten la misma morfología y sintaxis, incluso el mismo léxico. Es decir, sus diferencias fundamentales en muchos casos sólo afectan a la pronunciación (Younes 2006: 160-162). Por el contrario, la utilización de dariya implica un cambio mayor a todos los niveles.

Durante varios años, traté de comenzar la enseñanza del árabe, utilizando el dariya oralmente, pero enseñando la grafía del árabe estándar. Los resultados fueron decepcionantes, porque el uso de los dos registros producía confusión en los alumnos. Por ello, decidí abordar exclusivamente el dariya en el primer curso de LA, tanto a nivel oral como escrito. Lo que expongo a continuación es el resultado de mi experiencia y los materiales que he creado para llevarla a cabo, así como la justificación de cada una de las decisiones tomadas.

\section{Consideraciones previas}

\section{1. ¿Qué es la lengua árabe?}

Siempre se ha enseñado solo el árabe estándar, por tradición, motivos religiosos o estratégicos. Incluso para ampliar el posible mapa de comunicación, ya que es más atractiva una lengua con 300 millones de hablantes, que una con 30, como puede ser el árabe marroquí. Sin embargo, el problema fundamental que enfrenta la enseñanza de la LA es la distorsión que experimenta cuando se disocia una parte muy importante de ella. Comenzamos, pues, con una reflexión epistemológica.

Se ha explicado la realidad lingüística de la LA desde el prisma de la diglosia (Kaye 2001). Sin embargo, el árabe es algo más que una lengua diglósica. Se ha analizado también como un continuum lingüístico, con variantes y registros (Hary 1996). En cualquier caso, la LA comprende distintas variedades, que pueden ser incomprensibles entre sí. El bilingüismo, el multilingüismo el cambio de registro y la mezcla de registros es la realidad de la LA (Bassioney 2009 y Rosenhouse 2015). Es decir, el árabe clásico, la lengua oficial de los países de la Liga Árabe, no está solo, sino acompañado de todas y cada una de las variantes regionales de los hablantes nativos. No podemos entender la variante estándar sin al menos uno de los otros. Cuando se aborda la 
lengua considerando solo la variedad prestigiosa, se obtiene una visión incompleta de la misma que impide adquirirla y que crea mucha frustración en los estudiantes. Por mucho interés y esfuerzos que se le dediquen, no es posible ser competente en LA sin tener un dominio fluido de al menos una variedad oral $^{(1)}$.

Recientes investigaciones señalan, además, que al escuchar árabe estándar el cerebro de los nativos se comporta como como si se tratara de una lengua extranjera (Khamis-Dawkar y Froud 2007 y Khamis-Dawkar \& Makhloul 2014).

\subsection{Enseñanza de una L2 (Lengua Extranjera)}

Por otro lado, es importante aplicar a la LA todos los conocimientos de la enseñanza-aprendizaje de una L2. Para ello, es de obligada consulta el Marco Común Europeo de Referencia de Lenguas, que orienta sobre qué es una lengua y cómo enseñarla. El Marco no habla de diglosia, pero sí de multilingüismo. Y muchos de sus postulados nos ayudan a adoptar un criterio coherente en la enseñanza de la LA. Voy a fijarme en solo tres aspectos que apuntan a la importancia de adquirir al menos dos variantes.

a) Ámbitos de uso: tenemos que admitir que los registros empleados cambian considerablemente dependiendo de los ámbitos de uso de la lengua. Por ejemplo, el registro para pedir un vaso de agua en un bar o para abordar a alguien en la calle para pedir información no es el mismo que el que se emplea en una charla en una universidad. Si usamos árabe estándar en el primer caso, no estamos utilizando el registro lingüístico apropiado (que siempre sería regional, incluso de otro registro). Por tanto, la respuesta que obtenemos es acorde con nuestra equivocación: no conseguimos el vaso de agua o la información, sino una sonrisa burlona que apunta asombro, guasa o estupor. Esta respuesta revela que hemos infringido las reglas de la comunicación, al confundir el ámbito de uso, de modo que nuestra interacción no ha tenido éxito.

b) Necesidades de los alumnos: estas necesidades son un factor determinante para decidir el currículo de una materia y pueden variar a lo largo del tiempo.

(1) Federico Corriente ya apuntó la necesidad de conocer los dos registros en el prefacio a su gramática en 1980 (Corriente 1988: 11-14).

AAM, 26 (2019) 14.1-16 
En el mundo actual, las necesidades de los alumnos al aprender árabe son, sobre todo, ser capaz de comunicarse con los arabófonos de nuestro país, estar conectados al mundo árabe actual, a las redes sociales, conocer de cerca la inmigración, etc., mucho más que ser capaces de leer textos clásicos medievales.

c) Papel del profesor como facilitador: el buen profesor es capaz de guiar a los alumnos para adquirir la lengua meta, conforme a sus necesidades, teniendo en cuenta los ámbitos de uso de la lengua. Es decir, no es aquel que hace alarde de sus conocimientos gramaticales, sino el que es capaz de conectar con la necesidad del alumno y ayudarlo a progresar en su aprendizaje, incluso si para ello tiene que cambiar sus concepciones previas.

A nivel educativo, todas estas aportaciones nos permiten aseverar que cualquier materia denominada LA debería tratar necesariamente ambos registros de la lengua: el estándar escrito y el hablado. De lo contrario, no seremos fieles a su naturaleza y estaremos propiciando una disociación.

\subsection{El árabe en España}

La enseñanza del árabe en nuestro país ha estado vinculada con el arabismo, dentro del ámbito universitario, y con el africanismo, más afín al medio militar y religioso. Los estudios árabes han tenido gran tradición en España, acompañada por una extensa producción científica, más orientada a temas históricos que lingüísticos o didácticos, por lo que el aprendizaje de la LA se redujo a la transmisión del sistema e ignoró los registros orales. El africanismo, en cambio, se interesó por la lengua, en su variante dialectal más próxima: el marroquí, produciendo un buen número de gramáticas, diccionarios y manuales. De algún modo, esta situación reflejaba la dicotomía existente entre los registros lingüísticos -lengua normativa versus lengua hablada.

Actualmente, con más de 800.000 marroquíes censados en España, la LA es la segunda lengua extranjera de nuestro país. Además, hay que añadir la presencia cada vez mayor en las clases de LA de alumnos de herencia. Y las cifras van en aumento. Esto significa que la enseñanza del árabe en nuestro país debería incluir por fuerza el árabe marroquí. ¿Por qué no se hace así? Por dos motivos fundamentales: por el peso de la costumbre y por la falta de preparación del profesorado. El primer motivo argumenta que las cosas se han hecho 
siempre así, y quizá podamos encontrar un paralelismo con el cuento La punta del jamón ${ }^{(2)}$. La tradición no puede ser el principal argumento. Por lo que respecta a la falta de preparación del profesorado, es un problema que podría compararse con la dificultad de la paternidad responsable. La necesidad obligará a los docentes a ser conscientes de la necesidad de transformar el sistema. En el entorno educativo español, es evidente que serán los propios alumnos los que demanden la transformación.

\section{2. Árabe integrado: Dariya / fusha cara a cara}

Hemos visto que, desde un punto de vista pragmático, la naturaleza de la LA nos hace entender que cualquier materia denominada árabe o $L A$ debería tratar necesariamente sus dos registros: el estándar y el hablado. El modelo coherente de enseñanza de una lengua es aquel que recrea en el aula la situación real de la lengua. El hablante nativo pone en juego todas sus estrategias para realizar las tareas en las que necesita hacer un uso efectivo de su lengua. Y lo mismo debería darse en el caso del aprendiz. En este contexto, no es posible disociar el árabe normativo del darija, ni el darija del árabe normativo. Las competencias lingüísticas de uno y otro están entrelazadas porque su utilización depende de los distintos contextos comunicativos, así como de los temas y ámbitos específicos. Por lo que debería enseñarse al alumno las estrategias necesarias para elegir uno u otro registro dependiendo del contexto, ámbito o

(2) Se trata de una dramatización en tres actos de Thomas Harris, que ha sido adaptada en un cuento: J. Bucay (2012). Cartas para Claudia. RBA Libros.

(En la casa de una pareja, la esposa ha cocinado por primera vez jamón al horno).

Él: Está exquisito. ¿Para qué le cortaste la punta? Ella: El jamón al horno se hace así. Él: Eso no es cierto, yo he comido otros jamones asados enteros. Ella: Puede ser, pero con la punta cortada se cocina mejor. Él: ¡Es ridículo! ¿Por qué? Ella: Mi mamá me lo enseñó así. Él: ;Vamos a la casa de tu mamá!

(En la casa de la madre).

Ella: Mamá, ¿cómo se hace el jamón al horno? Madre: Se lo adoba, se le corta la punta y se lo mete al horno. Ella: (a Él) ¡¿Viste?! Él: Señora, ¿y para qué le corta la punta? Madre: ¡Mi madre me lo enseñó así! Él: ¡Vamos a la casa de la abuela!

(En la casa de la abuela).

Ella: Abuela, ¿cómo se hace el jamón al horno? Abuela: Lo adobo bien, lo dejo reposar tres horas, le corto la punta y lo cocino a horno lento. Madre/Ella: (a Él) ¿Viste? Él: Abuela, ¿para qué le corta la punta? Abuela: Hombre, jle corto la punta para me pueda entrar en la asadera! Mi horno es tan chico. 
tarea, del mismo modo que los utilizan sus hablantes. El aprendizaje y la enseñanza de la LA deberían abarcar necesariamente el registro oral-dialectal y el registro escrito-estándar. Su enseñanza debería responder a la realidad de la propia lengua, en la que ambos están ligados íntimamente.

Se ha argumentado que la enseñanza simultánea de una variante regional junto con el árabe estándar retrasa el aprendizaje. Quizá sólo es un mito. Lo cierto es que al menos no lo impide. La propuesta de árabe integrado responde a las necesidades del alumno, la elección del marroquí responde a la evidencia de la presencia marroquí en España y a la cercanía y relaciones con Marruecos.

\section{Nishan: material para iniciarse en el aprendizaje de la lengua árabe}

Después de más de veinte años de experiencia en elaborar materiales para aprender a leer y escribir en árabe, en colaboración con otros autores (Aguilar 1998, 2004, 2006, 2010, 2011, 2014 y 2019), decidí preparar un libro que unificara esta experiencia aplicándola a la enseñanza del registro oral del árabe marroquí. Para ello partí del material presentado en Alatul $^{(3)}$. El resultado final es نيشان / Nishan ${ }^{(4)}$. El libro está pensado para contextos docentes dirigidos, que puede usarse durante 50 o 60 horas de clase y que equivale a un nivel A1.1 del Marco. Para alcanzar este nivel en el tiempo propuesto, es fundamental que el profesor planifique los objetivos comunicativos que no se han desarrollado en detalle. También es recomendable que el alumno realice en casa de forma autónoma todos los ejercicios, salvo aquellos de discriminación oral.

Los criterios principales seguidos en la confección de Nishan son dos: utilizar la lengua siguiendo un enfoque comunicativo y seguir los presupuestos de la concepción constructivista del aprendizaje. Es decir, en cuando al orden de las letras a aprender se parte de lo fácil ( 0 , en la lección 1) para llegar a lo difícil (las enfáticas en la lección 8), por ejemplo; también se parte de aprender el nombre, y datos personales básicos, para aumentar los contenidos en complejidad. A lo largo de Nishan, se familiariza al alumno a leer y escribir en LA, utilizando el registro del árabe marroquí y asociando sonido con la grafía correspondiente.

(3) Este libro ha tenido ya cinco reimpresiones, lo cual de alguna forma demuestra que ha sido útil para introducir a los alumnos en la grafía de la LA.

(4) El término hace alusión al mismo significado de Alatul (recto, en línea recta) y también fue el nombre de una conocida revista marroquí (en francés Nichane), que se publicó entre 2006 y 2010 y fue promotora del uso del dariya (Aguadé 2012). 
La escritura del dariya tiene múltiples variantes (Caubet, 2017, Hoogland 2014, Miller 2017 y Moustaoui 2016) y todavía no está normalizada ${ }^{(5)}$. Nishan sigue la grafía más respetuosa con la LA estándar. Esta elección, aunque no se adecua a un criterio estrictamente fonético o habitual en dariya, está tomada por un criterio pedagógico, ya que facilita al alumno la capacidad de lectura en árabe estándar.

Se han mantenido, pues, algunas características del fusha, como, por ejemplo:

- La ausencia de vocales, que tienden a escribirse en marroquí.

- Consonantes que no existen en dariya, como las interdentales (ذ، ظ، ث).

- La escritura de los posesivos, pronombres personales o demostrativos

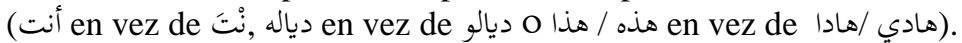

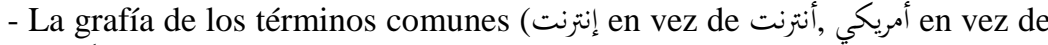
en vez de طليطالي o (طريكاني

- Algunas palabras que en marroquí han transformado las consonantes en enfáticas (دار en vez de ضار).

El libro tiene ocho lecciones, además de una introducción general sobre la LA y el árabe marroquí y otra lección de repaso. Cada lección está estructurada del mismo modo: tiene una parte teórica y otra práctica. En la parte teórica se presentan: una tabla con las nuevas letras, explicaciones sobre sus particularidades gráficas o fonéticas, algunos signos auxiliares, explicaciones gramaticales básicas, vocabulario para fijar las grafías, nombres propios frecuentes, léxico relacionado con algún área temática (saludos, familia, profesiones...) y expresiones comunes. La parte práctica está ordenada en dos bloques: ejercicios de lectura y ejercicios de escritura. En los primeros, hay ejercicios de discriminación auditiva (en palabras o frases), de reconocimiento de vocabulario familiar para el alumno, de búsqueda del intruso o de la frase correcta, así como de lectura. Los ejercicios de escritura tienden a fijar las letras aprendidas mediante la composición y descomposición de palabras, una planilla de caligrafía, así como pruebas sencillas de escritura de palabras aisladas o para completar frases o huecos, responder preguntas sencillas o conjugar verbos.

(5) También se puede escribir dariya en grafía latina, lo cual es muy común en móviles e internet (Caubet 2012).

AAM, 26 (2019) 14.1-16 
Los ejercicios están ordenados por su naturaleza (percepción o expresión). Pero no conviene hacerlos en el orden en el que se encuentran, sino intercalando unos con otros, de acuerdo a la interacción oral que se realiza en clase, acompañando las explicaciones gramaticales planteadas, de acuerdo a un criterio de menor a mayor complejidad.

Nishan presta especial atención a vocablos internacionales, nombres propios, topónimos y numerales, porque son muy rentables. El uso de topónimos y vocabulario que el alumno puede deducir con facilidad le ayudan a adquirir el nuevo código gráfico cómodamente, al centrar la tarea en la percepción visual, porque la auditiva es comprensible. No olvidemos que la lectura en árabe tiene una mayor complejidad que en español, porque, al tratarse de un código defectivo, mientras en nuestra lengua leemos para comprender, en árabe, si no se comprende no se puede leer ${ }^{(6)}$. Aunque la escritura en marroquí no es defectiva como en árabe estándar, el objetivo a largo plazo es facilitar que el alumno sea competente también en la lengua estándar; de lo contrario, estaríamos formando un analfabeto. En cuanto a los nombres propios y numerales, aunque son esenciales, no están exentos de dificultad. Concederles el tratamiento que se merecen, desde el primer momento y con la debida atención, supone que el alumno aborde su uso con naturalidad y le facilita el acceso a dos campos semánticos dispares respecto al español.

Para apoyar el material escrito, el libro está acompañado por noventa audios descargables en archivo mp3, con la correspondiente transcripción de los mismos. Se incluye también la clave de ejercicios. Todo ello para permitir al alumno trabajar de forma autónoma.

He de añadir que agradezco el trabajo de todos los compañeros que han publicado métodos para la enseñanza del árabe marroquí (Caubet 1993, Chekayri 2011, Moscoso et al. 2013), o diccionarios de esta lengua (el Ghazouani 2017 y Moscoso 2015). Sin duda, estas aportaciones me han inspirado para elaborar Nishan. Por ese motivo he incluido en el libro, que saldrá a la luz dentro de dos meses, referencias a estas y otras obras, aunque tal práctica no es frecuente en los materiales didácticos. Considero, sin embargo, que la adquisición del código gráfico de la LA no es, en absoluto, su dificultad mayor, como puedo comprobar año tras año en los resultados de mis alumnos,

(6) Un experimento neurofisiológico demuestra que hay más áreas del cerebro que participan en la tarea de «leer» en árabe que español (al-Hamouri et al. 2005). 
como voy a demostrar a continuación. Por ello, no creo que sea acertado enseñar marroquí en grafía latina, ya que de alguna forma también supone cierta disociación. Del mismo modo, la transcripción ni es rentable ni ayuda al alumno, porque es un artificio. Los estudiantes son capaces de adquirir el alfabeto árabe sin demasiada dificultad. En este sentido está dirigido Nishan, para orientar al profesor con detalle en presentar el alfabeto árabe, mientras que la parte oral, no menos importante, solo está apuntada, porque entiendo que es más intuitiva y fácil de realizar para el profesor.

\section{Resultados}

La experiencia de experimentar Nishan con los alumnos de $1^{\circ}$ curso (6 créditos: 60 horas lectivas), en los grados de Estudios Franceses, Estudios Ingleses, Lengua y Literatura Españolas y Traducción e Interpretación de la Universidad de Murcia, durante varios años ha sido muy satisfactoria. La mejor forma de ver los resultados obtenidos es por medio del sistema de evaluación, el cual está compuesto por varias pruebas:

- Un examen escrito, de cinco páginas, que incluye los siguientes ejercicios: una lectura, preguntas de verdadero/falso y preguntas cerradas sobre esta; frases para completar huecos, tablas con conjugaciones, deducir el significado, componer los gentilicios a partir de los topónimos, buscar el intruso o la frase correcta, reconocer la capital de algunos países árabes o el nombre de algunos signos auxiliares, unir y descomponer letras y palabras, formar expresiones.

- Varias audiciones: numerales, reconocimiento de palabras y frases, completar huecos en un texto.

- Una presentación escrita en dariya que incluya datos personales (nombre, apellido, edad, estudios, lenguas que se hablan, lugar de residencia, número de teléfono, nacionalidad, lugar de nacimiento, fecha de nacimiento, situación familiar y presentación de algún miembro de su familia, gustos, estatura y peso.

- Una presentación oral(7).

(7) La presentación oral siempre se graba con una cámara. Es similar a la escrita y no se puede leer, pero sí utilizar la presentación escrita como apoyo.

AAM, 26 (2019) 14.1-16 
Una imagen vale más que mil palabras. Por eso, presento a continuación los resultados de la utilización de Nishan durante el curso de Lengua C1: árabe, en el primer cuatrimestre del curso 2018/2019 del grado de Traducción e Interpretación. Incluyo la transcripción al árabe de una presentación oral, así como dos textos de presentaciones escritas. Agradezco a los alumnos la gentileza de haberme permitido presentar sus textos. He querido transcribir la presentación oral de uno de los alumnos para ver la diferencia entre esta y su presentación escrita. He intentado transcribir su pronunciación en la medida de lo posible. En el caso de no pronunciar algunas consonantes dobles, es difícil que se registre.

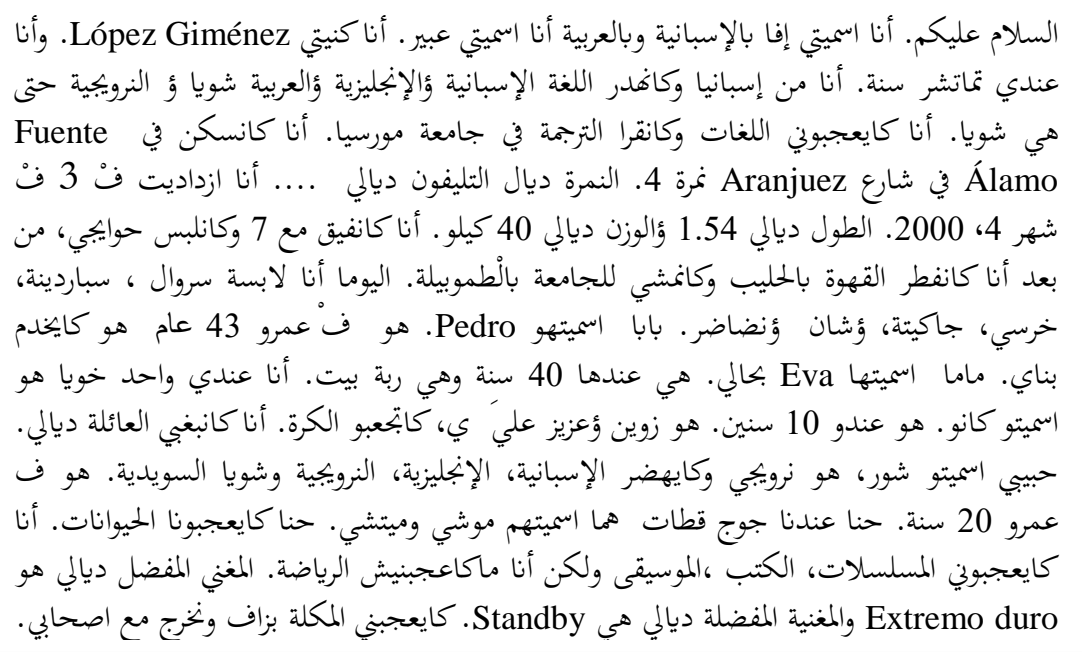

A continuación, está su presentación escrita:

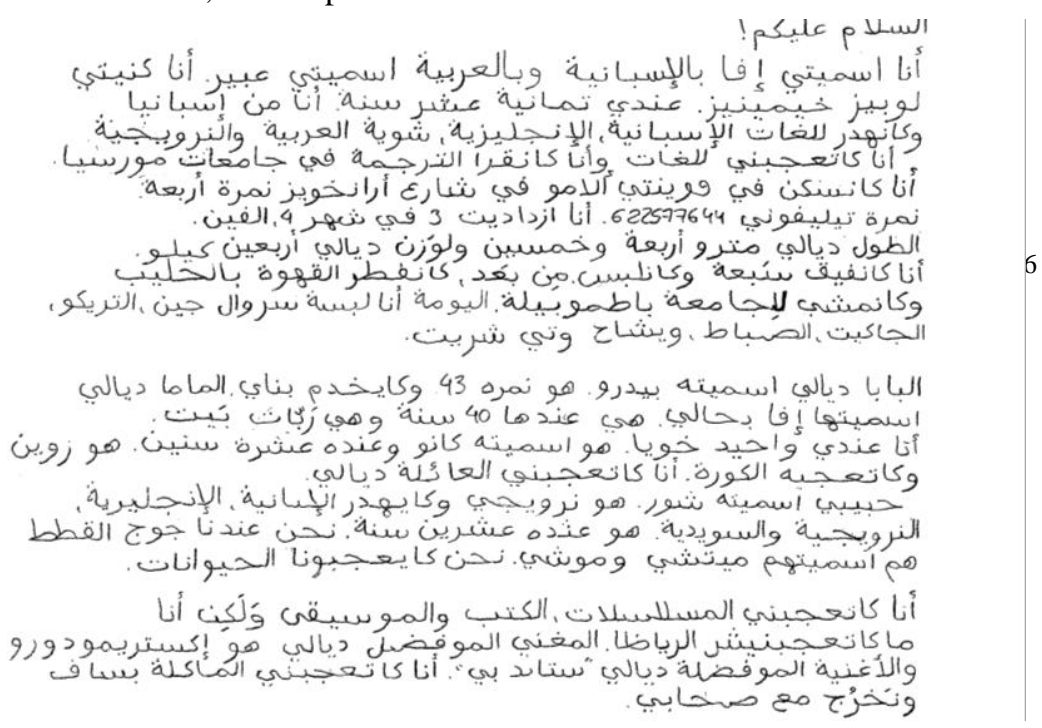


سلا مم ، كي داير؟ أنا اسميتي ميقيل كنيلي لو مبا ير ناند يس. أنا من

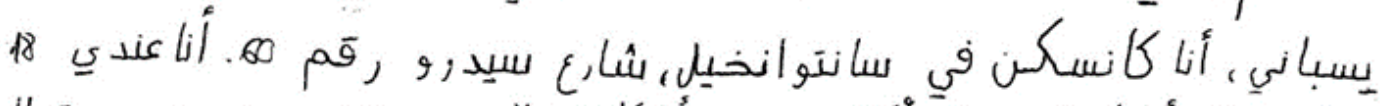

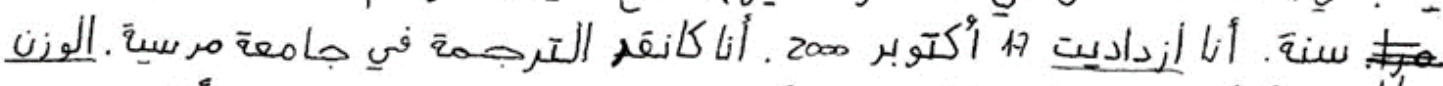

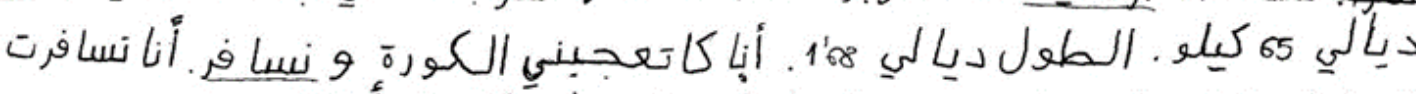

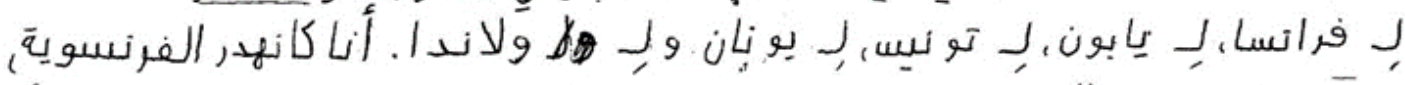

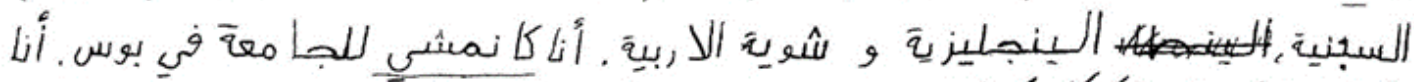

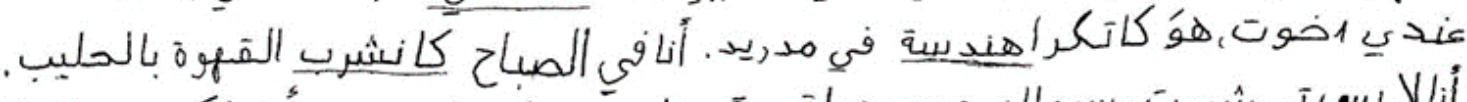

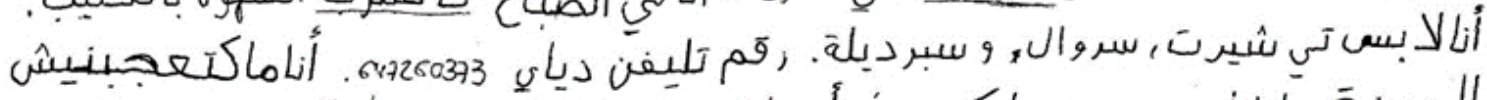

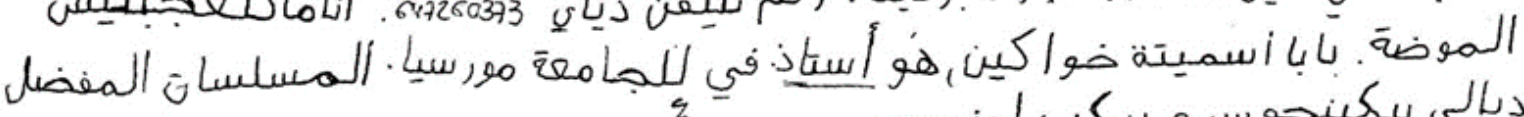

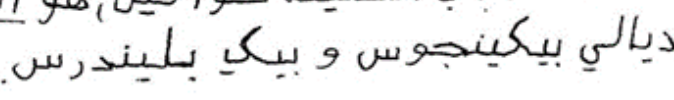

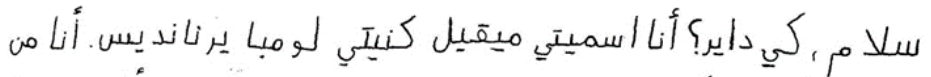

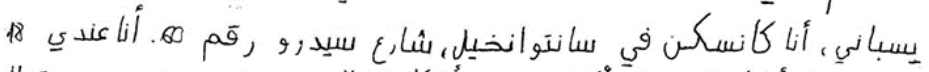

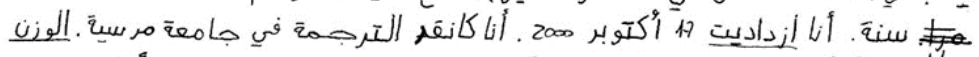

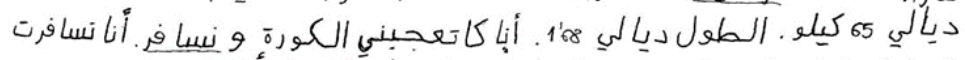

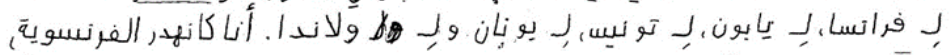

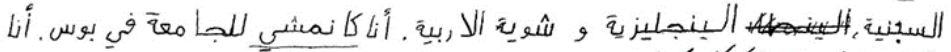

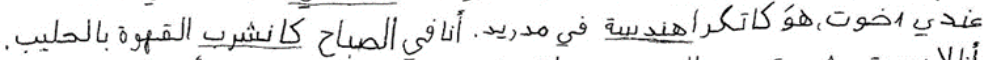

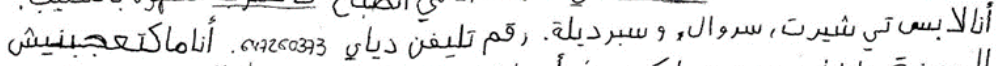

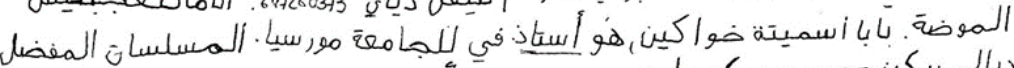

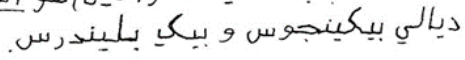




\section{BIBLIOGRAFÍA}

AGUADÉ, Jorge. 2012. «Monarquía, dialecto e insolencia en Marruecos: el caso de Nichane». En: M. Meouak, P. Sánchez \& Á. Vicente (eds.). De los manuscritos medievales a internet: la presencia del árabe vernáculo en las fuentes escritas. En: Colección Estudios de Dialectología Árabe 6. Zaragoza, Universidad de Zaragoza, pp. 441-464.

AGUILAR, Victoria. 2014. «Enseñanza conjunta del árabe moderno normativo y el marroquí». En: P. Santillán Grimm, L. M. Pérez Cañada \& F. Moscoso García (eds.). Árabe marroquí: de la oralidad a la enseñanza. Cuenca. Ediciones de la Universidad de Castilla-La Mancha, pp. 305-324.

AGUILAR, Victoria. 2019. Nishan. Iniciación al árabe marroquí. A1.1. Murcia, Diego Marín.

AGUILAR, Victoria \& FERNÁNDEZ FONFRIA, Lidia. 2016. «La conciencia lingüística de los estudiantes de árabe en España». En: F. Moscoso \& A. Moustaoui (eds.). Identidad y conciencia lingüística. VI Congreso de árabe marroquí. Madrid, UAM Ediciones, pp. 75-116.

AGUILAR, Victoria, GIMÉNEZ, Antonio, MANZANO, Miguel Ángel \& ZANÓN, Jesús. 2006. Paso a paso خطوة خطوة/ Libro electrónico en formato PDF, descargable en el repositorio de la Universidad de Alicante: <http://rua.ua.es/dspace/handle/10045/33513> [01 de noviembre de 2019].

AGUILAR, Victoria, MANZANO, Miguel Ángel y SEGURA Ma Dolores (2011), Alatul. Libro del profesor, Herdereditorial, Barcelona.

AGUILAR, Victoria, MANZANO, Miguel Ángel \& ZANÓN, Jesús. 1998. Hayyā natakallam al-'arabiyya. Cuaderno para leer y escribir árabe. Murcia, DM-ICE.

AGUILAR, Victoria, MANZANO, Miguel Ángel \& ZANÓN, Jesús. 2010. Alatul. Barcelona, Herder.

AGUILAR, Victoria, MANZANO, Miguel Ángel \& ZANÓN, Jesús. 2019. Letters and Meanings: Gateway to Arabic. Murcia, Editum. Libro electrónico en formato PDF, descargable en:

<https://libros.um.es/editum/catalog/book/2761> [01 de noviembre de 2019].

AGUILAR, Victoria, RUBIO, Ana \& DOMINGO, Lourdes. 2014. Mabruk. A2.1. Murcia, Diego Marín. 
AGUILAR, Victoria \& Zanón, Jesús 2004. Vocabulario básico árabe-español lespañol-árabe. Murcia, DM-ICE.

ALOSH, Mahdi. 1997. Learner, text, and context in foreign language acquisition: An Arabic perspective. Columbus, the Ohio State University National Foreign Language Resource Center.

AL-BATAL, M. \& BELNAP, R. K. 2006. «The Teaching and Learning of Arabic in the United States: Realities, needs, and future directions». En: K. M. Wahba, Z. A. Taha, \& L. England (eds.). Handbook for Arabic Language Teaching Professionals. Nueva Yersey, Lawrence Erlbaum, Mahwah, pp. 389-399.

BASSIOUNEY, Reem. 2009. Arabic sociolinguistics. Topics in Diglossia, Gender, Identity and Politics. Edimburgo, Edinburgh University Press.

BENÍTEZ, Monserrat. 2012. «TelQuel: una fuente contemporánea para el estudio del árabe marroquí». En: M. Meouak, P. Sánchez \& Á. Vicente (eds.). De los manuscritos medievales a internet: la presencia del árabe vernáculo en las fuentes escritas. En: Colección Estudios de Dialectología Árabe 6. Zaragoza, Universidad de Zaragoza, pp. 403-418.

CAUBET, Dominique. 1993. L'arabe marocain. 2 vols. Paris, Peeters.

CAUBET, Dominique. 2012. «Apparition massive de la darija à l'écrit à partir de 2008-2009 : sur le papier ou sur la toile : quelle graphie ? Quelles régularités ?». En: M. Meouak, P. Sánchez \& Á. Vicente (eds.). De los manuscritos medievales a internet: la presencia del árabe vernáculo en las fuentes escritas. En: Colección Estudios de Dialectología Árabe 6. Zaragoza, Universidad de Zaragoza, pp. 377-402.

CAUBET. D. (2017). Morocco an informal passage to literacy in darija (Moroccan Arabic). En: Hoigilt. J, Mejdell. G. (eds.). The politics of Written language in the Arab World. Leiden, Brill. pp. 116-142.

CORRIENTE, Federico. 1988. Gramática árabe. Barcelona, Herder.

CHEKAYRI, Abdellah. 2011. An introduction to Moroccan Arabic and culture. Georgetown. Georgetown University Press.

EL GHAZOUANI, Lahoucine. 2017. Diccionario español-árabe marroquí, AlMadyaq. Madrid, Editum-Universidad de Murcia.

AL-HAMOURI, $\mathrm{F}$ et al. 2005. «Brain dynamics of Arabic reading: A magnetoencephalographic study». NeuroReport 16, pp. 1861-1864.

HARY, B. 1996. «The language continuum in Arabic multiglossia». En: A. Elgibali (ed.). Understanding Arabic, Essays in Contemporary Arabic 
Linguistics in Honor of El-Said Badawi. El Cairo, the American University in Cairo Press, pp. 69-90.

HOOGLAND, Jan. 2014. «Towards a standardized orthography of Moroccan Arabic based on best practices and common ground among a selection of authors». En: P. Santillán Grimm, L.M. Pérez Cañada \& F. Moscoso García (eds.). Árabe marroquí: de la oralidad a la enseñanza. Cuenca, Ediciones de la Universidad de Castilla-La Mancha, pp. 59-76.

HORN, C. 2015. «Diglossia in the Arab World. Educational Implications and Future, Perspectives» Open Journal of Modern Linguistics 5, pp. 100-104. $<10.4236 /$ ojml.2015.51009> [01 de noviembre de 2019]

KAYE, A. S. 2001. «Diglossia: The State of the Art» International Journal of the Sociology of Language 152, pp. 117-129.

KHAMIS-DAKWAR, Reem \& FROUD, Karen. 2007. «Lexical processing in two language varieties: An event-related brain potential study of Arabic native speakers». En: M. Mughazy (ed.). Perspectives on Arabic Linguistics XX: Papers from the Twentieth Annual Symposium on Arabic Linguistics, Kalamazoo, Michigan, March 2006. Amstercam, John Benjamins, pp. 153-166.

KHAMIS-DAKWAR, Reem \& MAKHOUL, B. 2014. «Arabic diglossia and its implications for language learning disability assessment». En: E. SaeighHaddad \& M. Joshi (eds.). Handbook of Arabic Literacy: Insights and Perspectives. Amsterdam-Nueva York, Springer, pp. 279-303.

KHAMIS-DAKWAR, Reem \& FROUD, Karen (en prensa): «The study of Arabic language acquisition». En: K. Ryding, K. \& D. Wilmsen (eds.). The Cambridge Handbook of Arabic Linguistics. Cambridge, Cambridge University Press.

Marco Común europeo de referencia para las lenguas: aprendizaje, enseñanza, evaluación (2002). Madrid, Consejo de Europa, Instituto Cervantes. <http://cvc.cervantes.es/obref/marco/> [01 de noviembre de 2019].

MILLER. C. 2017. «Contemporary darija writings in Morocco. Ideology and practices». En: Hoigilt. J, Mejdell. G. (eds.). The politics of Writte language in the Arab World. Leiden, Brill. pp. 90-116.

MOSCOSO GARCÍA, Francisco. NOUAOURI IZRELLI, Nadi Hamdi \& RODRÍGUEZ GARCÍA, Óscar. 2013. B chuiya b chuiya: Al. Árabe marroquí. Almería, Albujayra. 
MOSCOSO GARCÍA, Francisco. 2015. Diccionario de árabe marroquí. Gijón, Trea.

MOUSTAOUI SRHIR. A. (2016). «Writing in Moroccan Arabic as a linguistic practice: diversity and linguistic heterogeneity». Chapter 7, En: Moustaoui Srhir, Adil, Sociolinguistics of Moroccan Arabic: new topics. Frankfurt\&Wien, Peter lang. pp. 103-115.

PALMER, J. 2007. «Arabic diglossia: Teaching only the standard variety is a disservice to students». The Arizona Working Papers in Second Language Acquisition and Teaching 14, pp. 111-122.

ROSENHOUSE, Judith. 2015. «Bilingualism/Multilingualism in the Middle East and North Africa: A Focus on Cross-National and Diglossic Bilingualism/Multilingualism». En: T. K. Bhatia y W.C. Ritchie (eds.). The Handbook of Bilingualism and Multilingualism. Chichester, Wiley Blackwell, pp. 899-919.

YOUNES, Munther. 2006. «Integrating the Colloquial with Fuṣhà in the Arabic-as-a-Foreign-Language Classroom». En: K.M. Wahba, Z.A. Taha \& L. England (eds). Handbook for Arabic Language Teaching Professionals in the 21st century. Mahwa (Nueva Jersey), Lawrence Erlbaum, pp. 157-166.

YOUNES, Munther. 2014. The integrated approach to Arabic instruction. Londres-Nueva York, Routledge.

VERSTEEGH, Kees. 2006. History of Arabic language teaching. En: W. M. Kassem, Z. A. Taha \& L. England (eds). Handbook for Arabic Language Teaching Professionals in the 21st century. Mahwa (Nueva Jersey), Lawrence Erlbaum, pp. 3-12.

VERSTEEG, Kees. 2014². The Arabic Language. Edimburgo, Edimburgh University Press.

WAHBA, Kassem M. 2006. «Arabic Language Use and the Educated Language User». En: K. M. Wahba, Z. A. Taha \& L. England (eds.). Handbook for Arabic Language Teaching Professionals in the 21st century. Mahwa (Nueva Jersey), Lawrence Erlbaum, pp. 139-155.

WAHBA, Kassem M., TAHA, Zeinab A. \& ENGLAND, Liz (eds.). 2006. Handbook for Arabic Language Teaching Professionals in the $21^{\text {th }}$ century. Mahwah (Nueva Jersey), Lawrence Erlbaum. 